\title{
Automated Infrared Pupillometer Use in Assessing the Neurological Status in Pediatric Neurocritical Care Patients: Case Reports and Literature Review
}

\author{
Molly E. McGetrick ${ }^{1}$ Nathan Schneider ${ }^{2,3}$ DaiWai M. Olson ${ }^{3,4}$ Venkatesh Aiyagari ${ }^{3,4}$ \\ Darryl Miles ${ }^{10}$
}

${ }^{1}$ Division of Critical Care, Department of Pediatrics, The University of Texas Southwestern Medical Center, Dallas, Texas, United States

2 The University of Texas Southwestern ODL Brain Institute, Dallas, Texas, United States

${ }^{3}$ Department of Neurology, The University of Texas Southwestern Medical Center, Dallas, Texas, United States

${ }^{4}$ Department of Neurosurgery, The University of Texas Southwestern Medical Center, Dallas, Texas, United States

Address for correspondence Molly E. McGetrick, MD, Division of Critical Care, Department of Pediatrics, The University of Texas Southwestern Medical Center, 5323 Harry Hines Blvd Dallas TX, 75390, United States

(e-mail: molly.mcgetrick@Utsouthwestern.edu).

J Child Sci 2021;11:e125-e132.

\begin{abstract}
Keywords

- Pediatrics

- neurocritical care

- neurological monitoring

- cardiac arrest

- brain injury

Automated infrared pupillometry (AIP) is rapidly becoming an accepted standard for the evaluation of pupil size and reactivity in adult neurocritical care. Recently, pediatric centers are increasingly utilizing this technology, but data supporting its use in children are limited. Our pediatric intensive care unit instituted AIP as a standard of care for pupillary light assessments in neurocritical care patients in early 2020. In this article, we describe four cases highlighting the advantage of using objective assessments of the pupillary light reactivity response measured by the Neurological Pupil index (NPi) to detect early changes in the patient's neurological status. These cases support the applicability of AIP in pediatric neurocritical care as a noninvasive neurologic monitoring tool. The NPi may be superior to manual pupil assessments by providing a numerical scale for accurate trending clinical status of a patient's neurologic condition.
\end{abstract}

\section{Introduction}

An estimated $16 \%$ of all pediatric intensive care unit (PICU) admissions are secondary to acute neurologic illness and while technological advances have improved medical care leading to lower mortality rates, adverse outcomes due to neurocritical illnesses remain high. Pediatric patients with neurologic diagnoses have significantly higher mortality rates and percentages of severe long-term neurologic morbidities than the general PICU population. ${ }^{1}$ For instance, the age-adjusted mortality rate is more than doubled $(5.7$ per 100,000$)$ in children with traumatic brain injury (TBI) compared with those with childhood cancer $\left(2.0\right.$ per 100,000). ${ }^{2,3}$ Despite the seriousness of

received

January 22, 2021

accepted after revision

March 31, 2021 these conditions, neurological monitoring remains largely dependent on serial clinical neurologic exams. The pupillary light reflex (PLR), which involves constriction and relaxation of the iris sphincter muscle in response to an external light stimulus, is an essential component of the neurologic examination and is particularly consequential in states of coma or altered sensorium where other components of the neurological exam are less discernible. Loss of the PLR can indicate increased intracranial pressure (ICP), midbrain dysfunction, or horizontal or vertical shifts in brain parenchyma due to mass effect. Until recently, only manual methods for assessing pupillary reactivity were available that used imprecise descriptors such as "brisk," "sluggish" or "absent" to describe the PLR,

\section{(c) 2021. The Author(s).}

This is an open access article published by Thieme under the terms of the Creative Commons Attribution License, permitting unrestricted use, distribution, and reproduction so long as the original work is properly cited. (https://creativecommons.org/licenses/by/4.0/)

Georg Thieme Verlag KG, Rüdigerstraße 14, 70469 Stuttgart, Germany 
introducing a potential for bias and error. A study of manual bedside pupil assessments found remarkable variation from provider to provider with interexaminer discrepancies occurring in $>40 \%$ of assessments and a $50 \%$ failure rate for detecting clinically significant anisocoria. ${ }^{4}$

Automated infrared pupillometry (AIP) was developed to decrease interobserver variability and create objective output measured variables of the PLR in responses to a calibrated standard light source. ${ }^{5}$ This advance greatly improved upon traditional methods for assessing PLR by removing the subjective components of pupillary size, reactivity to variable light stimuli. ${ }^{6}$ The pupillometer is a hand-held computer-based infrared digital video camera device that measures pupil size to within $0.01 \mathrm{~mm}$ and quantifies components of PLR including the percent change and velocity of constriction from baseline following light stimulus, the latency or time of onset of the constriction response, and dilation velocity or the speed of dilation recovery after the constriction response. The Neurological Pupil index (NPi) is an algorithmic score combining the AIP features of the PLR into a numerical value ranging from 0 to 5 , where 0 indicates a nonreactive pupil, a value less than 3 indicates an abnormal response, and a value of 3 or greater is considered normal. The automated pupillometry exam takes approximately 3 seconds to perform and has been studied in children of all ages without any adverse events, although it may be limited by patient cooperation in awake children. ${ }^{7}$ Studies in adult patients support a potential role of the NPi assessed by AIP for prognostication after cardiac arrest and subarachnoid hemorrhage, ${ }^{8-12}$ detection of significant intracranial events, ${ }^{13-15}$ and elevated ICP, ${ }^{16-20}$ and for detecting nonconvulsive status epilepticus. ${ }^{21,22}$ Published literature established normative data PLR reference values in healthy children, but investigations of AIP use in neurocritically ill pediatric patients are limited to a single study on its use for detection of increased ICP. ${ }^{7,23}$ This report highlights four cases where trend changes in NPi values served as a valuable objective measure for detection of neurological worsening and acute neurological events in pediatric neurocritical illness (-Table 1).

\section{Case 1: In-Hospital Cardiac Arrest}

A previously healthy 2-year-old presented to the emergency department (ED) unresponsive in shock secondary to acute vomiting, diarrhea, and sepsis. Despite resuscitative efforts in the ED the patient became pulseless and cardiopulmonary resuscitation (CPR) was initiated. Return of spontaneous circulation (ROSC) was achieved after 20 minutes of CPR and inotropic medications were started for hemodynamic support. In the PICU, the patient remained hemodynamically unstable despite increasing inotropic support and was cannulated to venoarterial extracorporeal membrane oxygenation. After cannulation, AIP was ordered to coincide with her neurologic assessments. Throughout the first 22 hours spontaneous movement of all extremities was noted with intact brainstem reflexes and bilateral NPi values greater than 4 (-Fig. 1, Case 1). Between hours 22 and 29, intermittent drops in her NPi from a baseline of 4.8 bilaterally to a minimum of 3.2 on the right and 3.4 on the left were observed without significant anisocoria.
Changes in the NPi were correlated with onset of seizure activity on electroencephalogram (EEG) and the NPi returned to baseline once seizures were controlled with antiepileptic medications. An increase in pupil size was also observed over this time period. Between hours 40 and 45 after PICU admission, a downward trend in the right NPi to an abnormal value of 2.6 was noted, while the left NPi remained at 4.7. Her neurological exam performed after anisocoria was noted at hour 45 revealed absence of withdrawal response to painful stimuli and loss of corneal, cough, and gag reflexes. Mannitol was administered followed by a brief return of the right NPi to a baseline value of 4.7, although the remainder of the clinical neurological exam was otherwise unchanged. The pupils rapidly thereafter became less reactive as indicated by the fall in the NPi (right then left) and by hour 56, bilateral values were 0 . A head computed tomography (CT) revealed diffuse cerebral edema, basal cistern effacement, and uncal herniation ( - Fig. 2A). Given her poor neurologic prognosis, mechanical circulatory support was withdrawn, and the patient expired.

\section{Case 2: Out-of-Hospital Cardiac Arrest}

A previously healthy 17-year-old was found unresponsive at home. At the scene emergency medical services noted agonal breathing and a palpable pulse. The patient subsequently became pulseless with a pulseless electrical activity rhythm and CPR was started. After 25 minutes of CPR, ROSC was obtained. The initial blood gas in the ED demonstrated a significant metabolic acidosis with a $\mathrm{pH}$ of 6.9 , a $\mathrm{pCO}_{2}$ of 43 , and a base deficit of 24 . Other notable laboratory values were a blood glucose of greater than $700 \mathrm{mg} / \mathrm{dL}$, elevated hepatic transaminases, and acute kidney injury. Further history revealed a 2-week history of weight loss, polydipsia, polyuria, and fatigue consistent with new-onset diabetic ketoacidosis. Upon arrival to the PICU, hourly AIP was ordered with his neurological assessments. Notably, he had anisocoria-the right pupil measured $6.5 \mathrm{~mm}$ and left measured $4.6 \mathrm{~mm}$ with bilateral normal NPi values of 4.5 (-Fig. 1, Case 2). A head CT was obtained shortly after PICU admission which did not show any acute intracranial abnormality (-Fig. 2B). Despite receiving no sedative or paralytic medications, the patient lacked spontaneous eye opening or response to painful stimuli for the initial 24 hours, although cough and gag reflexes were present. The course was complicated by idioventricular rhythm requiring lidocaine infusion and hypotension requiring three continuous vasoactive infusions (epinephrine, norepinephrine, and vasopressin). The NPi readings remained stable and above 4.0 bilaterally during the admission, consistent with normally reactive pupils ( - Fig. 1, Case 2). Insulin was titrated to correct the hyperglycemia with an improving neurologic exam improved, and vasoactive infusions were discontinued. On hospital day (HD) 7 the patient was transferred out of the PICU and a brain magnetic resonance imaging (MRI) study on HD 10 was normal. The patient was discharged home without any residual neurological deficit.

\section{Case 3: Vertebral Artery Dissection and Cerebellar Stroke} A previously healthy 14-year-old presented with 2 weeks of morning headache which on the day of presentation was 
Case 1 Cardiac Arrest
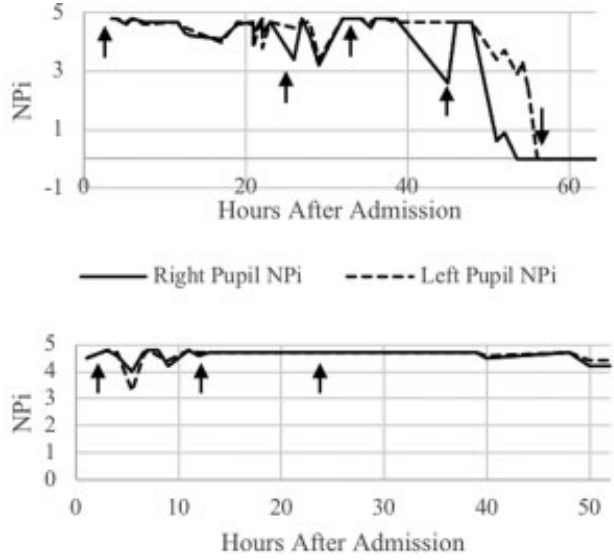

Case 2 Cardiac Arrest

Case 3 Cerebellar Stroke

Case 4 Traumatic Subdural

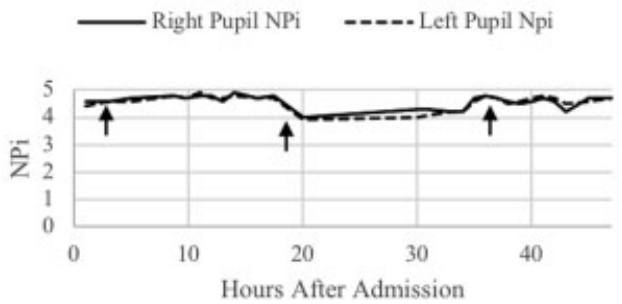

- Right Pupil NPi -----. Left Pupil Npi

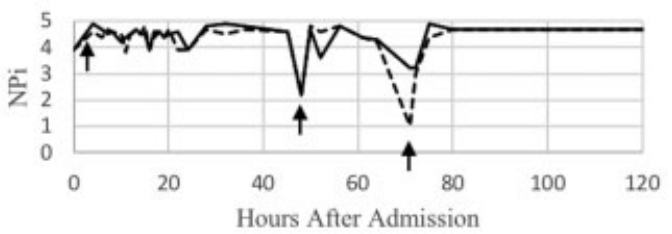

R pupil NPI -----L L pupil NPI

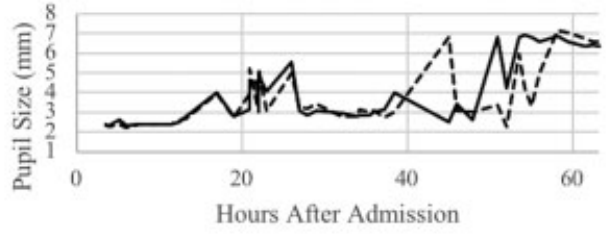

— Right Pupil Size $\quad$------ Left Pupil Size

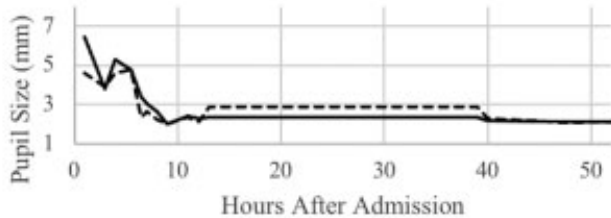

_ Right Pupil Size ------ Left Pupil Size

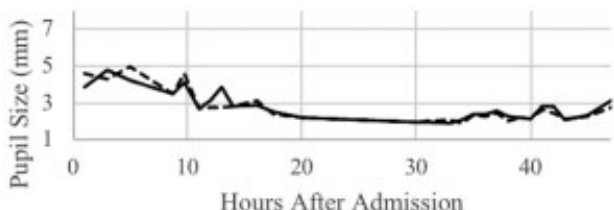

— Right Pupil size $\quad$-.--.- Left Pupil sizc

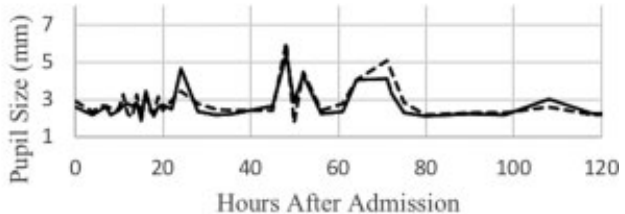

$-R$ pupil size $\quad$----.-L $L$ pupil size

Fig. 1 A graphic representation of Neurological Pupil index (NPi) and pupil size reported over time for cases 1-4. Case 1: NPi values immediately following emergent cannulation to venoarterial (VA) ECMO established a baseline of 4.8 . Between hours 22 and 29 electrographic seizure activity is associated with decreases in NPi values to 3.4, which return to baseline following treatment of seizures by hour 32 . At hour 45 , a significant fall in the right pupil NPi to 2.6 occurs with loss of cough and gag reflexes and absence of withdrawal to painful stimulus. Mannitol (1 g/ $/ \mathrm{kg})$ is administered followed by brief improvement in NPi. Between hours 50 and 55, bilateral NPi values (right then left) show a rapid decline to zero associated with increase in pupil size. The patient continues to show poor neurological function and a CT scan at hour 56 reveals diffuse cerebral edema and uncal herniation. Case 2: admission PICU NPi values of 4.8 in a comatose state. There is a brief fall in the left NPi that occurs after admission, which remains above 3 and quickly returns to baseline values and remain above 4.5. At hour 12 the patient exhibits withdrawal response to painful stimuli but otherwise remained unresponsive. By hour 24 the patient begins to make purposeful movements and follow simple commands. Case 3: baseline NPi values upon PICU admission of 4.7. Between hours 18 and 20 the NPi drops by 0.8 points bilaterally to 3.9 over 2 hours that persists. The patient then began exhibiting speech difficulties followed by facial droop prompting imaging and emergent occipital decompressive craniotomy and extraventricular drain (EVD) placement. Patient returns to PICU from operating room with improvement in NPi values to previous baseline of 4.5-4.7. Case 4: baseline NPi values upon PICU admission of 3.9-4.7 At hour 48, the patient experiences clinical seizure activity, associated with an intracranial pressure spike to $25 \mathrm{~mm} \mathrm{Hg}$ and a drop in the NPi to 2.2 bilaterally. By hour 50 , clinical seizure activity resolved, and EEG was consistent with absence of electrographic seizures. Again, at hour 71, there was a decline in the NPI to 3.2 on the right and 1.0 on the left associated with subclinical seizures localized to the right frontal region. These abnormal discharges resolved by hour 74 and the NPi returned to baseline of 4.7. CT, computed tomography; EEG, electroencephalogram; PICU, pediatric intensive care unit.

unrelenting. Initial vital signs showed hypertension with a systolic blood pressures above $150 \mathrm{~mm} \mathrm{Hg}$ and bradycardia with a heart rate less than 50 beats per minute. On neurological examination, a left-sided dysmetria, decreased tone in the left-upper and left-lower extremities, and a wide-based gait were found. Neuroimaging revealed a left vertebral artery dissection with associated nonocclusive thrombus and an acute left posterior-inferior cerebellar artery territory infarction. The patient was admitted to the
PICU for close neurological monitoring and initiation of systemic anticoagulation with heparin. Pupillometry was ordered on admission, which established a normal baseline $\mathrm{NPi}$ of 4.2 to 4.9 . Although headaches persisted, no new neurologic symptoms developed in the first 18 hours of PICU admission. Between hours 18 and 20, there was an acute drop in the bilateral NPi values by 0.8 (-Fig. 1, Case 3). By hour 22, the patient became confused with incomprehensible speech, and a new left-sided facial droop was found. 

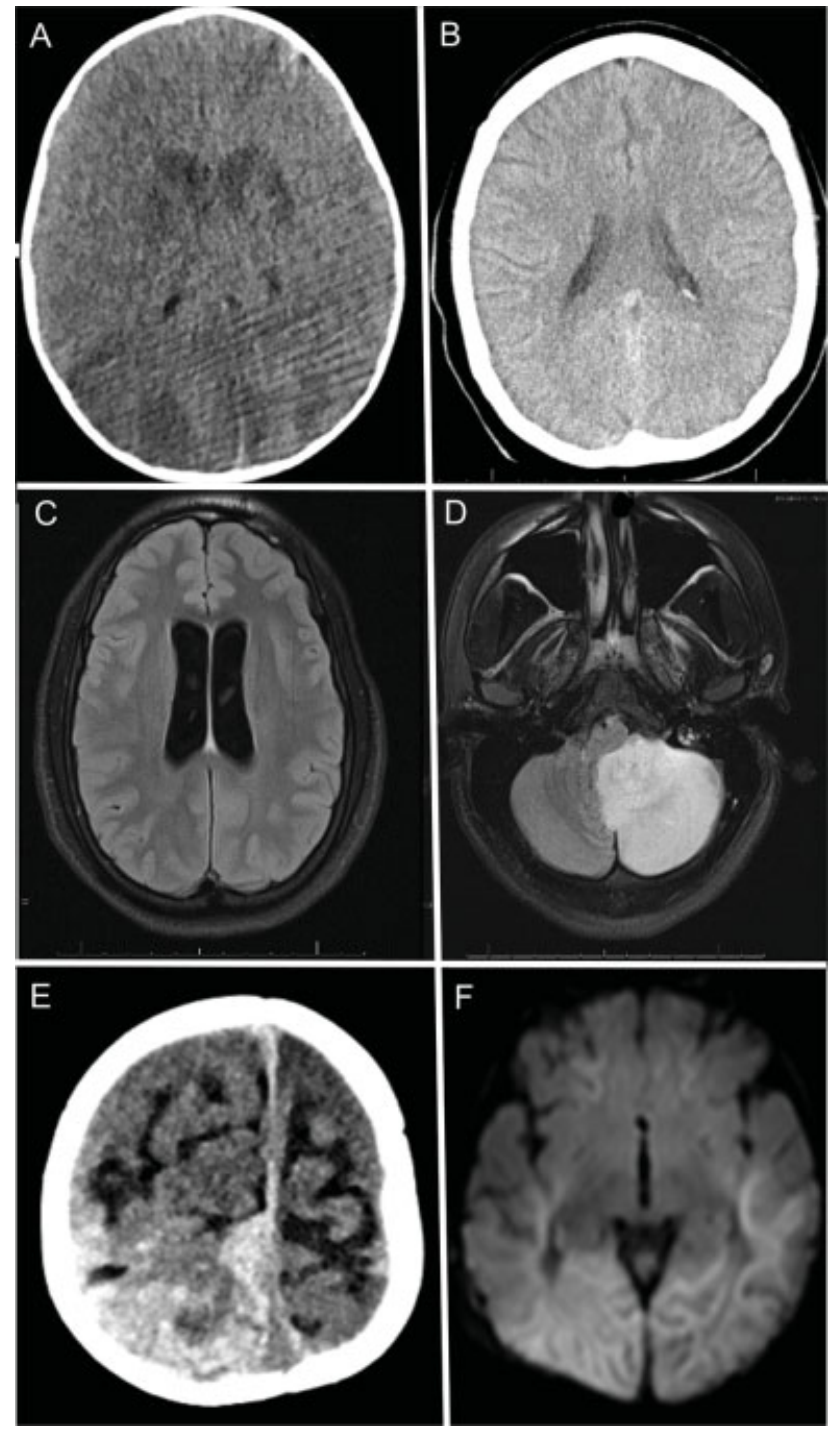

Fig. 2 Neuroimaging in cases 1-4. Axial brain computed tomography (CT) scan obtained at hour 56 in case 1 demonstrating diffuse cerebral edema with effacement of basal cisterns, cerebral sulci, and bilateral uncal herniation (A). Admission axial brain CT scan obtained in case 2 with no evidence of acute intracranial abnormality (B). Axial magnetic resonance imaging (MRI) sequence obtained at hour 21 of admission in case 3 showing developing hydrocephalus (C) secondary to compression of the fourth ventricle due to mass effect from acute left-sided cerebellar infarct (D). Hospital admission axial brain CT image showing acute right subdural hemorrhage (E). Brain MRI diffusion-weighted image on hospital day 3 demonstrates extensive bilateral hemispheric hypoxic-ischemic injury (F).

A repeat MRI showed developing obstructive hydrocephalus related to increased left cerebellar swelling and mass effect on the fourth ventricle ( - Fig. 2C). A ventriculostomy catheter was placed and the patient underwent emergent posterior fossa decompressive craniectomy. Immediately after returning from the operating room, the NPi values returned to their baseline values near 4.5 . The ventricles remained adequately decompressed on repeat neuroimaging and the patient underwent cranioplasty on HD 14 and was discharged to in-patient rehabilitation with improving ataxia and mild leftsided weakness.

\section{Case 4: A Traumatic Subdural Hematoma}

A 9-month-old female with no significant past medical history presented to the ED after a fall from a bed at home. The initial Glasgow Coma Score was 4 with extensor posturing and no spontaneous eye opening. She was emergently intubated and presumed intracranial hypertension was treated with 3\% hypertonic saline and $60 \mathrm{mg} / \mathrm{kg}$ of intravenous (IV) levetiracetam for seizure prophylaxis. A head CT demonstrated a large right-sided subdural hemorrhage and a nondepressed occipital skull fracture. In the PICU, an ICP monitor was placed with an opening pressure of $6 \mathrm{~mm} \mathrm{Hg}$. Initially, the pupils measured $2.6 \mathrm{~mm}$ on the right and $2.9 \mathrm{~mm}$ on the left by AIP with an NPi of 3.9 and 4.5 , respectively. Over the first 48 hours, the patient was sedated on fentanyl and midazolam infusions with an ICP below $20 \mathrm{~mm} \mathrm{Hg}$. At hour 48 she developed acute tachycardia and upper extremity twitching associated with elevated ICP to $25 \mathrm{~mm}$ Hg. Simultaneously, her bilateral NPi dropped from 4.5 to 2.2 bilaterally and her pupil size increased from 2.4 to $6 \mathrm{~mm}$ (-Fig. 1, Case 4). Electrographic seizures were confirmed on bedside EEG and treated with increasing midazolam infusion. Brain MRI revealed diffuse signal changes consistent with cytotoxic edema from hypoxic-ischemic injury (-Fig. 2F). At hour 71 the patient exhibited another acute drop from a baseline NPi of 4-4.5 to 3.2 on the right and 1 on the left eye, which was associated with an elevated ICP spike to $34 \mathrm{~mm} \mathrm{Hg}$. The patient was paralyzed at this time, and seizure activity was noted on the EEG. IV lacosamide was administered and by hour 74 , all seizure activity stopped and the subsequent NPi values remained stable $(>4.0)$ for the duration of the monitoring period. ICP remained low, and the patient was extubated on HD 10 and later discharged to inpatient rehabilitation with residual left-sided motor impairments and paroxysmal sympathetic hyperreactivity.

\section{Discussion}

In January 2020, our institution changed the method of assessing pupil size and reaction to include objective assessments of the PLR with a NeurOptics NPi-200 pupillometer (Laguna Hills, California, United States) in all children admitted to the PICU following a TBI, cardiac arrest, stoke, intracranial hemorrhage, or with any neurocritical illness at risk for neurological decline. The cases described in this report illustrate potential advantages of using AIP as a noninvasive neuromonitoring tool in the neurological assessment of critically ill pediatric patients (-Table $\mathbf{1}$ ). The NPi as a quantifiable measurement of the PLR provides an objective trend to detect changes in the PLR compared with traditional standard manual assessments limited to subjective descriptions such as "brisk," "reactive," or "sluggish." Similar to findings published in adult populations, we found that changes in the NPi may be a useful marker for early detection of significant neurologic events including brain herniation, convulsive and nonconvulsive seizures, and hydrocephalus. ${ }^{5,9,10,13,21,24}$

In cases 1 and 2, we demonstrate that pupillometry may provide valuable information regarding the development of significant brain swelling and herniation during the postarrest phase of care. In case 1 , we present a young child who 


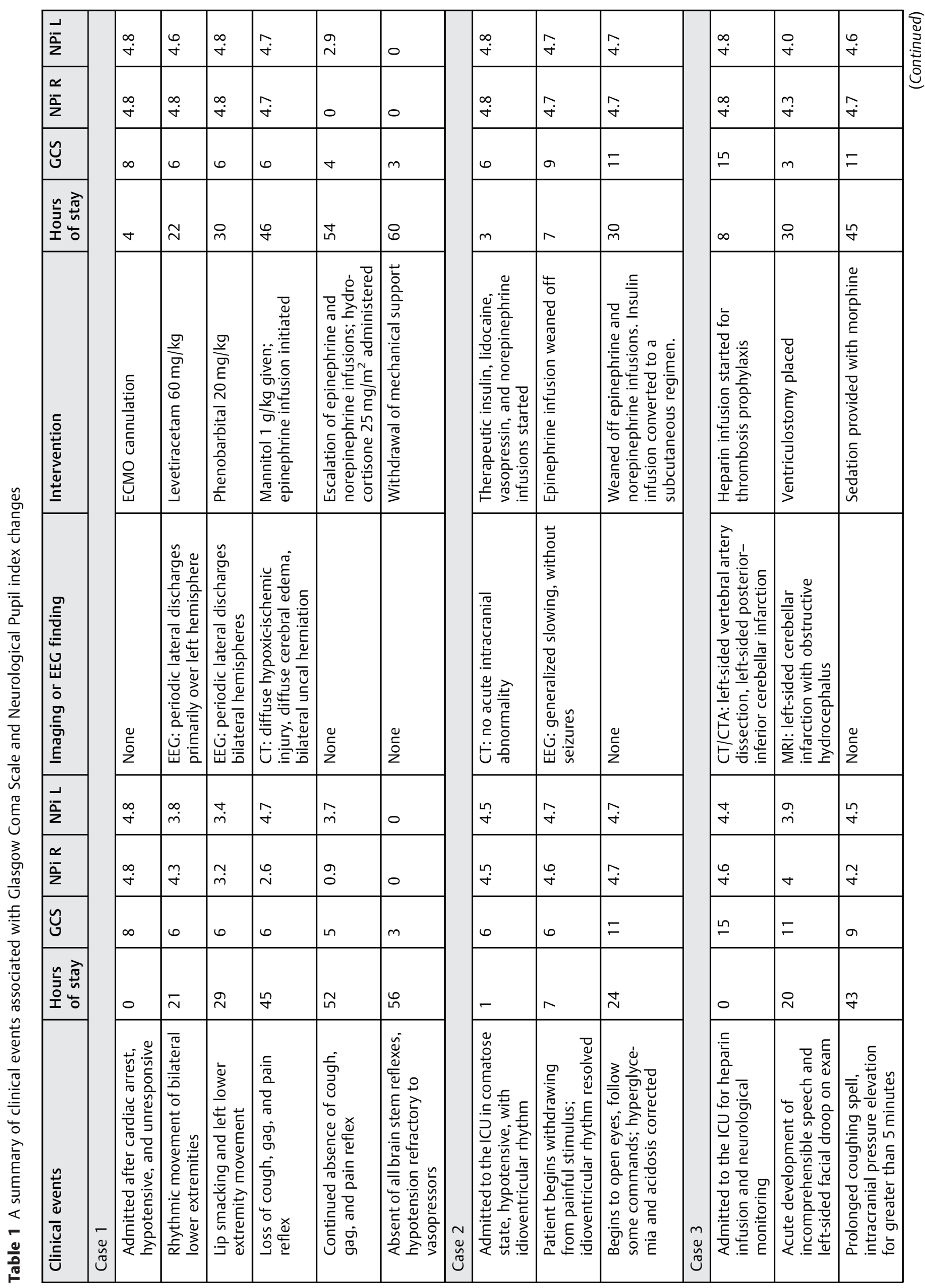




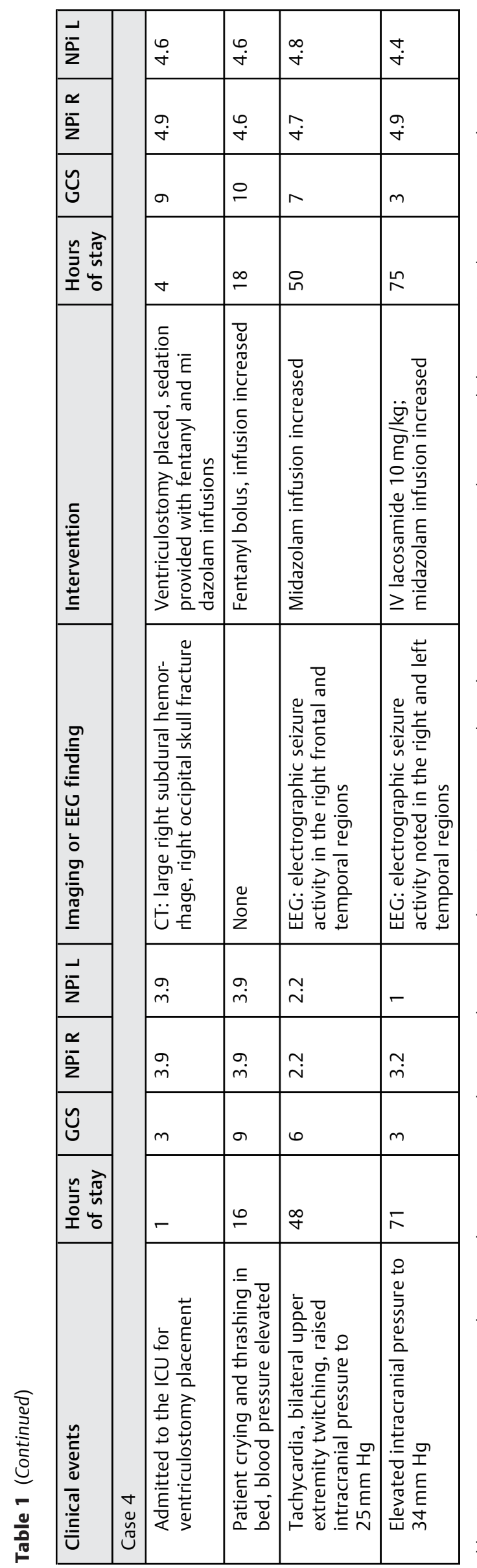

experienced an in-hospital cardiac arrest and despite reported normal pupillary reactivity ( $\mathrm{NPi}>3$ ) for much of the course, downward trends in the patient's NPi preceded documented clinical neurological changes. The continued decline in the patient's NPi to 0 ultimately correlated with progressive cerebral edema and herniation with complete loss of the PLR and neurological function. Although in-hospital cardiac arrests are associated with higher likelihood of survival compared with those that occur outside the hospital, mortality is still high. Recent evidence from the Get With The GuidelinesResuscitation registry, a United States-based in-hospital cardiac arrest registry, reported that $62 \%$ of children who arrest within the hospital will die, with brain death accounting for greater than half of these deaths. ${ }^{25}$ Despite implementation of evidence-based neuroprotective strategies in the critical hours after cardiac arrest, neurological injury from anoxic insults may progress leading to cerebral swelling, neuronal cell death, and brainstem herniation, as seen in the unfortunate demise of case 1 . Although the patient suffered a more abrupt fall in the NPi and rapid clinical deterioration, changes in the PLR detected by AIP may occur prior to changes in the clinical exam when significant intracranial events occur. In one report, changes in NPi occurred at a median of 7.4 hours before transtentorial herniation occurred. ${ }^{15}$ This is demonstrated in case 3 where we see a fall in NPi hours before a clinical change was observed. In herniation events with a more insidious onset, AIP may uncover subtle changes in pupillary reactivity much earlier than a standard clinical examination would allow, providing opportunity for life-saving intervention. In case 2 the patient suffered an out-of-hospital cardiac arrest of similar duration to case 1 and presenting physical and laboratory examinations might suggest a poor neurologic prognosis. In contrast to case 1 where the patient had a declining NPi within 48, the patient in our second case had bilateral NPi values that remained normal despite an initial comatose state, and as his neurological status gradually improved in the following days. Because the clinical exam was limited by coma, the PLR and NPi values were important clinical markers used at the bedside to assess this patient's neurological status. Recent adult studies have suggested that AIP may serve as a prognostication tool after cardiac arrest, with reasonable associations between abnormal NPi and mortality or unfavorable neurologic outcome. ${ }^{8-10}$ Although there may be stark differences in the etiologies for cardiac arrest in children compared with adults, our experiences suggest that API could potentially play a role in prognostication after cardiac arrest in children as well.

In cases 3 and 4, a pupillometry NPi decline trend was associated with evolving neurological changes in the patient's condition. In case 4 a decline in the NPi was associated with an increase in ICP levels above 20, coinciding with onset of seizures. The relationships between the NPi and invasive ICP values have been explored in adult patients as a noninvasive method for detecting elevated ICP. ${ }^{17,18,20}$ In a pediatric prospective observational study by Freeman et al, 1,130 paired measurements of invasive ICP and NPi values were analyzed in 28 children with invasive ICP monitors placed secondary to either TBI or encephalopathy. ${ }^{23}$ The 
authors found a modest but significant inverse correlation ( -0.31 [95\% confidence interval: -0.53 to -0.1 ],$p=0.004$ ) between the NPi and ICP values. The reliability of using NPi for noninvasively detecting intracranial hypertension is still being elucidated with a need for more robust datasets investigating this relationship in children of all ages. In case 3 the downward trend in NPi which persisted below the patient's previously established baseline was associated with developing hydrocephalus and raised ICP that was ultimately amendable surgical or medical intervention resulting in a good outcome.

Interestingly, in cases 1 and 4 declining NPi was associated with convulsive and electrographic seizure activity, which improved with appropriate antiepileptic therapy. The association between seizures and changes in the NPi has until recently not been well described, and to our knowledge the existing literature is limited to adults. A recent study conducted in adult patients demonstrated that nonconvulsive status epilepticus was associated with significant reductions in NPi from baseline, and discrepancies between right and left NPi during an event. ${ }^{21}$ Our findings suggest that exploration of changes in AIP in patients with epileptic events could pose an additional area of investigation.

It is worth noting that although the manufacturer suggests an NPi threshold of 3 to distinguish between normal and abnormal pupillary reactivity, serially trend values over time in patients may also provide important information for evolving neurologic injury. In the study by Freeman et al, a significant difference in mean NPi values (4.4 versus 3.4) was found when the ICP was less than or greater than 20 respectively $(p<0.001)$, although in both cohorts a value greater than 3 would be considered normal. In case 3 a persistent change in bilateral NPi values occurred by 0.8 from a baseline of 4.7 to 3.9 , which was associated with clinical worsening and developing hydrocephalus. In case 1 declines in NPi which still remained above 3 were associated with seizure activity. Therefore, a single NPi value might represent a clinically relevant change which could be interpreted as normal (above the 3 threshold) without establishing baseline AIP values. In our PICU pupillometer policy, medical staff are to be notified if the NPi falls to less than 3 or if there is a change of $>1$ that does not return to baseline within 30 minutes, recognizing that a fall from 4.5 to 3.1 may be clinically meaningful even though the absolute value is still above 3 .

There is a growing body of literature supporting AIP use in adult patients for applications described in these cases, nevertheless literature in pediatrics is scare. This series was designed to describe a small number of representative cases where AIP provided noninvasive detection of neurologic changes in the clinical status of pediatric patients with acute brain injury. Given this small sample size, as well as single-center design, our experiences may not be shared by all pediatric centers using this device for patients with brain injury. Although these cases suggest that pupillometry trends may provide valuable detection of significant neurologic events in the setting of known or suspected brain injury, further investigations are needed to elucidate situations in which measurements from this device may be limited.
Combining the experience of multiple institutions would certainly add awareness about the benefits of AIP and may help elucidate sources of error.

\section{Conclusion}

Automated pupillometry can provide valuable clinical information as a noninvasive neurologic monitoring tool in pediatric patients with acute neurological injury who are at risk for neurologic events. This technology may detect changes in the PLR not observable with manual pupil assessments providing earlier detection allowing for more timely intervention to reduce secondary brain injury. The use of automated pupillometry for the detection of subclinical seizures, increased ICP, and injury prognostication in children are interesting potential applications of this technology and deserve further study in children.

\section{Conflict of Interest}

None declared.

\section{Acknowledgments}

The authors would like to thank the excellent nurses at the University of Texas Southwestern and Children's Medical Center for their hard work in implementing automated pupillometry, and for their intense dedication to patient care.

\section{References}

1 Fink EL, Kochanek PM, Tasker RC, et al; Prevalence of acute critical neurological disease in children: a Global Epidemiological Assessment (PANGEA) Investigators. International survey of critically ill children with acute neurologic insults: the prevalence of acute critical neurological disease in children: a global epidemiological assessment study. Pediatr Crit Care Med 2017;18(04): 330-342

2 Miller KD, Fidler-Benaoudia M, Keegan TH, Hipp HS, Jemal A, Siegel RL. Cancer statistics for adolescents and young adults, 2020. CA Cancer J Clin 2020;70(06):443-459

3 Cheng P, Li R, Schwebel DC, Zhu M, Hu G. Traumatic brain injury mortality among U.S. children and adolescents ages $0-19$ years, 1999-2017. J Safety Res 2020;72:93-100

4 Olson DM, Stutzman S, Saju C, Wilson M, Zhao W, Aiyagari V. Interrater reliability of pupillary assessments. Neurocrit Care 2016;24(02):251-257

5 Phillips SS, Mueller CM, Nogueira RG, Khalifa YM. A systematic review assessing the current state of automated pupillometry in the NeuroICU. Neurocrit Care 2019;31(01):142-161

6 Lee MH, Mitra B, Pui JK, Fitzgerald M. The use and uptake of pupillometers in the intensive care unit. Aust Crit Care 2018;31 (04):199-203

7 Shah SS, Ranaivo HR, Mets-Halgrimson RB, Rychlik K, Kurup SP. Establishing a normative database for quantitative pupillometry in the pediatric population. BMC Ophthalmol 2020;20(01):121

8 Riker RR, Sawyer ME, Fischman VG, et al. Neurological pupil index and pupillary light reflex by pupillometry predict outcome early after cardiac arrest. Neurocrit Care 2020;32(01):152-161

9 Obling L, Hassager C, Illum C, et al. Prognostic value of automated pupillometry: an unselected cohort from a cardiac intensive care unit. Eur Heart J Acute Cardiovasc Care 2020;9(07):779-787

10 Oddo M, Sandroni C, Citerio G, et al. Quantitative versus standard pupillary light reflex for early prognostication in comatose cardiac 
arrest patients: an international prospective multicenter doubleblinded study. Intensive Care Med 2018;44(12):2102-2111

11 Mader MM, Piffko A, Dengler NF, et al. Initial pupil status is a strong predictor for in-hospital mortality after aneurysmal subarachnoid hemorrhage. Sci Rep 2020;10(01):4764

12 Ortega-Perez S, Shoyombo I, Aiyagari V, et al. Pupillary light reflex variability as a predictor of clinical outcomes in subarachnoid hemorrhage. J Neurosci Nurs 2019;51(04):171-175

13 Weerakoon SM, Stutzman SE, Atem FD, Kuchenbecker KS, Olson DM, Aiyagari V. Investigation of pupillary changes after carotid endarterectomy and carotid stent placement using automated pupillometry. J Stroke Cerebrovasc Dis 2020;29(05):104693

14 Kim TJ, Park SH, Jeong HB, et al. Neurological pupil index as an indicator of neurological worsening in large hemispheric strokes. Neurocrit Care 2020;33(02):575-581

15 Papangelou A, Zink EK, Chang WW, et al. Automated pupillometry and detection of clinical transtentorial brain herniation: a case series. Mil Med 2018;183(1-2):e113-e121

16 Al-Obaidi SZ, Atem FD, Stutzman SE, Olson DM. Impact of increased intracranial pressure on pupillometry: a replication study. Crit Care Explor 2019;1(10):e0054

17 McNett M, Moran C, Grimm D, Gianakis A. Pupillometry trends in the setting of increased intracranial pressure. J Neurosci Nurs 2018;50(06):357-361

18 Stevens AR, Su Z, Toman E, Belli A, Davies D. Optical pupillometry in traumatic brain injury: neurological pupil index and its rela- tionship with intracranial pressure through significant event analysis. Brain Inj 2019;33(08):1032-1038

19 Chen JW, Gombart ZJ, Rogers S, Gardiner SK, Cecil S, Bullock RM. Pupillary reactivity as an early indicator of increased intracranial pressure: The introduction of the Neurological Pupil index. Surg Neurol Int 2011;2:82

20 Jahns FP, Miroz JP, Messerer M, et al. Quantitative pupillometry for the monitoring of intracranial hypertension in patients with severe traumatic brain injury. Crit Care 2019;23(01):155

21 Godau J, Bierwirth C, Rösche J, Bösel J. Quantitative infrared pupillometry in nonconvulsive status epilepticus. Neurocrit Care 2020. Doi: 10.1007/s12028-020-01149-1

22 Hasan S, Peluso L, Ferlini L, et al. Correlation between electroencephalography and automated pupillometry in critically ill patients: a pilot study. J Neurosurg Anesthesiol 2021;33(02):161-166

23 Freeman AD, McCracken CE, Stockwell JA. Automated pupillary measurements inversely correlate with increased intracranial pressure in pediatric patients with acute brain injury or encephalopathy. Pediatr Crit Care Med 2020;21(08):753-759

24 El Ahmadieh TY, Bedros N, Stutzman SE, et al. Automated pupillometry as a triage and assessment tool in patients with traumatic brain injury. World Neurosurg 2021;145:e163-e169

25 Holmberg MJ, Wiberg S, Ross CE, et al. Trends in survival after pediatric in-hospital cardiac arrest in the United States. Circulation 2019;140(17):1398-1408 